OPEN ACCESS

Edited by:

Jordi Pérez-Tur,

Superior Council of Scientific

Investigations (CSIC), Spain

Reviewed by:

Doyoun Kim,

Korea Research Institute of Chemical Technology (KRICT), South Korea

Myriam Srour,

McGill University, Canada

${ }^{*}$ Correspondence:

Yuanlin Ma

mayuanlin2005@163.com

Xuefeng Wang

xfyp@163.com

Xin Tian

xintian@cqmu.edu.cn

Specialty section:

This article was submitted to Genetic Disorders,

a section of the journal

Frontiers in Genetics

Received: 19 August 2019 Accepted: 17 January 2020

Published: 27 February 2020

Citation:

Guo $Y$, Chen $Y$, Yang $M, X u X, L$ in $Z$, Ma J, Chen $H$, Hu Y, Ma Y, Wang $X$ and Tian X (2020) A Rare KIF1A

Missense Mutation Enhances

Synaptic Function and

Increases Seizure Activity.

Front. Genet. 11:61.

doi: 10.3389/fgene.2020.00061

\section{A Rare KIF1A Missense Mutation Enhances Synaptic Function and Increases Seizure Activity}

\author{
Yi Guo ${ }^{1}$, Yuanyuan Chen ${ }^{1}$, Min Yang ${ }^{1}, X i n X u^{1}$, Zijun Lin ${ }^{1}$, Junhong $\mathrm{Ma}^{2}$, \\ Hongnian Chen ${ }^{1}$, Yida Hu ${ }^{1}$, Yuanlin Ma ${ }^{1 *}$, Xuefeng Wang ${ }^{1 *}$ and Xin Tian ${ }^{1 *}$ \\ 1 Department of Neurology, The First Affiliated Hospital of Chongqing Medical University, Chongqing Key Laboratory of \\ Neurology, Chongqing, China, ${ }^{2}$ Center of Epilepsy, Beijing Institute for Brain Disorders, Beijing, China
}

Although genetic factors are considered a main etiology of epilepsy, the causes of genetic epilepsy in the majority of epilepsy patients remain unknown. Kinesin family member $1 \mathrm{~A}$ (KIF1A), a neuron-specific motor protein that moves along with microtubules, is responsible for the transport of membranous organelles and synaptic vesicles. Variants of KIF1A have recently been associated with hereditary spastic paraplegia (HSP), hereditary sensory and autonomic neuropathy type 2 (HSANII), and intellectual disability. However, mutations in KIF1A have not been detected in patients with epilepsy. In our study, we conducted customized sequencing of epilepsy-related genes of a family with six patients with generalized epilepsy over three generations and identified a rare heterozygous mutation (c.1190C > A, p. Ala397Asp) in KIF1A. Whole-cell recordings from primary cultured neurons revealed that the mutant KIF1A increases the excitatory synaptic transmission but not the intrinsic excitability of neurons, and phenotype testing in zebrafish showed that this rare mutation results in epileptic seizure-like activity. These results provide new evidence demonstrating that KIF1A dysfunction is involved in epileptogenesis.

Keywords: KIF1A, epilepsy, synaptic plasticity, dendritic spine, epileptogenesis

\section{INTRODUCTION}

Genetic factors are one of the main causes of epilepsy that have been confirmed by the International League Against Epilepsy (ILAE) (Berg et al., 2010; Scheffer et al., 2016). In 1995, Steinlein et al. demonstrated that missense mutations in CHRNA4 can cause autosomal dominant nocturnal frontal lobe epilepsy (Steinlein et al., 1995). A survey of 1,258 patients with epilepsy (of which 958 had focal epilepsy) showed that common gene variants collectively explain at least $26 \%$ of the phenotypic variation among patients with all forms of epilepsy and $27 \%$ of that among patients with focal epilepsy (Speed et al., 2014). Miller et al. analyzed possible factors affecting epilepsy in twins and found that genetic factors play a significant role in various types of seizures (Miller et al., 1998). Genetic epilepsy is defined as a direct result of a known or presumed genetic defect, and recurrent seizures are the core symptom of these disorders (Scheffer et al., 2016). With the development of next-generation sequencing techniques, significant progress toward understanding the genetic mechanisms underlying the disease has recently been achieved. However, the genetic etiology of epilepsy in most patients remains unknown. 
KIF1A is responsible for the transport of membranous organelles and synaptic vesicles in neurons, and mutations in KIF1A have been associated with hereditary spastic paraplegia (HSP), hereditary sensory and autonomic neuropathy type 2 (HSANII), and intellectual disability (Riviere et al., 2011; Citterio et al., 2015). Only a handful of previous reports have related KIF1A gene mutations to epileptic seizure, and thus far, the role of KIF1A in the origin of epilepsy has received limited attention.

In our study, we identified a rare mutation of KIF1A in a family with six patients over three generations who presented with generalized epilepsy. First, we found that the mutant KIF1A increased the excitatory synaptic density, and a functional test of zebrafish transfected with wild-type (WT) and mutant kiflaa using the tol 2 vector showed that the mutation caused epileptic seizure-like behavior and epileptic electrophysiological activity.

\section{MATERIALS AND METHODS}

\section{Genetic Screening of Epilepsy Patients}

DNA was extracted from peripheral blood lymphocytes collected from all individuals belonging to the family of interest. We performed customized sequencing of epilepsy-related genes (Supplementary Table 1) using NextSeq500 (Illumina, San Diego, CA, USA) to identify a possible causative gene mutation. Single nucleotide variations, insertions and deletions were then examined using GATK (https://software. broadinstitute.org/gatk/). Variants annotated in ANNOVAR (http://annovar.openbioinformatics.org/en/latest/) and variants with a minor allele frequency of at least 0.05 were filtered out (the candidate normal population database included 1000 Genomes, Exome Variant Server and EXAC). PolyPhen-2, SIFT and MutationTaster and GERP++ were used to predict the damage caused by all candidate variants, and the candidate causative variants obtained from the sequencing analysis were also confirmed by Sanger sequencing.

\section{Construction of Plasmids}

Flag-tagged human WT KIF1A was obtained by subcloning the complementary DNA (cDNA) into the pcDNA3.1-3xFlag-T2AEGFP plasmid using primers:

\section{F: CTTGGTACCGAGCTCGGATCCGCCACCATGGCCGGG GCTTCGGTGAA \\ R: GAAGGGCCCTCTAGACTCGAGGACCCGCATCTGG GCAGACC}

The missense mutation 1190C > A (p. Ala397Asp) was constructed by overlap PCR mutagenesis using the following primers:

KIF1A A397D-1F, CTTGGTACCGAGCTCGGATCCGCC ACCATGGCCGGGGCTTCGGTGAA;

KIF1A A397D-1R, CCCACCAGGtCATTGGTCATGTC AGTGATGTCGC;
KIF1A A397D-2F, ATGACCAATGaCCTGGTGGGT ATGAGCCCCTCAT; and

KIF1A A397D-2R, GAAGGGCCCTCTAGACTCGAGGAC CCGCATCTGGGCAGACC.

\section{Culture and Transfection of Primary Hippocampal Neurons}

The procedure used for culturing primary hippocampal neurons was previously described (Yang et al., 2019). Briefly, hippocampal neurons were prepared from C57BL/6 mouse embryos at gestational day 18 . The brains were removed, and the hippocampi were dissected from the brains. The obtained tissue was digested using $3 \mathrm{ml}$ of trypsin solution for $15 \mathrm{~min}$. The obtained neurons were cultured in neurobasal medium supplemented with B27, L-glutamine, penicillin, and streptomycin. The neurons were plated onto glass coverslips coated with poly-D-lysine in a $37^{\circ} \mathrm{C}$ incubator with $5 \% \mathrm{CO}_{2}$. The neurons were transfected with KIF1A-WT or KIF1A-A397D plasmids using the calcium phosphate transfection method at 7 days in vitro (DIV7).

\section{Whole-Cell Recordings}

Whole-cell recordings were obtained from green fluorescent protein (GFP)-positive neurons transfected with KIF1A-WT or KIF1A-A397D plasmids. To explore the intrinsic excitability of the neurons, we applied a depolarizing current of $500 \mathrm{~ms}$ in the current clamp mode starting from $-30 \mathrm{pA}$ and at increments of $10 \mathrm{pA}$ to induce action potentials. The rheobase was defined as the first current step that was able to induce action potential firing in a neuron. For the evaluation of synaptic transmission, we maintained the neurons at the potential of $-70 \mathrm{mV}$ in artificial cerebrospinal fluid (ACSF) containing $140 \mathrm{mM} \mathrm{NaCl}$, $5 \mathrm{mM} \mathrm{KCl}, 1.8 \mathrm{mM} \mathrm{CaCl}_{2}, 1.2 \mathrm{mM} \mathrm{MgCl}_{2}$, and $10 \mathrm{mM} \mathrm{D}$ glucose. The miniature excitatory post-synaptic currents (mEPSCs) were recorded in the presence of $1 \mu \mathrm{M}$ tetrodotoxin (TTX) and $0.1 \mathrm{mM}$ picrotoxin (PTX), and the miniature inhibitory post-synaptic currents (mIPSCs) were recorded in the presence of $1 \mu \mathrm{M}$ TTX, $10 \mu \mathrm{M}$ 6-cyano-7-nitroquinoxaline2,3-dione (CNQX), and $100 \mu \mathrm{M}$ (2R)-amino-5phosphonovaleric acid (APV). Electrophysiological data were acquired using a Multiclamp 700B amplifier and Digidata 1440A, and the data were analyzed using Mini Analysis (Synaptosoft, Leonia, NJ, USA) and Clampfit 10.3.

\section{Western Blot and Immunofluorescence Staining}

For western blotting, total protein was obtained from primary cultured hippocampal neurons 3 days after transfection with the KIF1A-WT or KIF1A-A397D plasmid using the calcium phosphate transfection method at DIV7. The western blot analysis was performed as described previously (Zhang et al., 2019), antibodies using for western blot including: mouse antiFlag (Sigma), Goat anti-mouse IgG (Proteintech), rabbit antiGAPDH (Proteintech), Goat anti-rabbit IgG (Proteintech).

For immunofluorescence staining, the cultured neurons were fixed with $4 \%$ paraformaldehyde/4\% sucrose in phosphate- 
buffered saline (PBS) for $40 \mathrm{~min}$ and permeabilized with $0.3 \%$ Triton X-100 in PBS for 15 min. Primary antibodies were diluted and added to the coverslip, and incubated overnight in a humidified chamber at $4^{\circ} \mathrm{C}$, then coverslips were washed three times with PBS and incubated with the secondary fluorescent antibodies at room temperature for $1 \mathrm{~h}$. Images were captured using a confocal laser scanning microscope (Leica, Wetzlar, Germany). The following primary antibodies were used: rabbit anti-GFP (Invitrogen), Guinea pig anti-vGLUT (synaptic systems), mouse anti-PSD-95 (Cell Signaling Technology), mouse anti-Flag (Sigma). The following secondary antibodies were used: Alexa Fluor 488-conjugated goat anti rabbit IgG (Invitrogen), Alexa Fluor 405-conjugated goat anti Guinea pig IgG (Jackson ImmunoResearch), Alexa Fluor 594-conjugated goat anti mouse IgG (Invitrogen).

For the assessment of neuronal morphology, the neurons were transfected with the KIF1A-WT or KIF1A-A397D plasmid at DIV7 and fixed at DIV10. In Sholl analysis, concentric circles were drawn around the soma every $10 \mu \mathrm{m}$, and the intersections with neurite branches were counted, then primary and secondary neurites in the same neurons were then counted.

For the analysis of spine density, the neurons were transfected with the KIF1A-WT or KIF1A-A397D plasmid at DIV7 and fixed at DIV16. The spine density was then quantified from two randomly selected secondary or tertiary dendrites per neuron. All these experiments were performed in a blinded manner by two observers.

\section{Zebrafish Maintenance and Breeding}

Adult male and female zebrafish (Danio rerio) of the $\mathrm{AB}$ strain were obtained from the China Zebrafish Resource Center (http:// www.zfish.cn/) and maintained at $28.5^{\circ} \mathrm{C}$ under a 14 -h light/10-h dark cycle using standard procedures. The fertilized eggs were collected via natural spawning. The fertilized embryos were maintained in medium containing $1.5 \mathrm{mM}$ 4-(2-hydroxyethyl)1-piperazineethanesulfonic acid (HEPES), $\mathrm{pH} 7.6,17.4 \mathrm{mM}$ $\mathrm{NaCl}, 0.21 \mathrm{mM} \mathrm{KCl}, 0.12 \mathrm{mM} \mathrm{MgSO}_{4}$, and $0.18 \mathrm{mM} \mathrm{Ca}(\mathrm{NO} 3)$ ${ }_{2}$ at $28.5^{\circ} \mathrm{C}$. All the experiments with zebrafish were approved by the Ethics Committee of Chongqing Medical University.

\section{Overexpression Experiments}

WT zebrafish kif1aa cDNA was cloned into the Tol2 expression vector (a gift from Koichi Kawakami, National Institute of Genetics) using following primers:

\section{F: AAAGAATTCCTCGACGGATCCGCCACCatggcaggg gcctcggtg;}

R: TCACCATGGTGGCGACCGGTCCAGAGCCTCCAC CCCCaaacctcatctgccagc;

The mutation encoding A433D in zebrafish was cloned into the kiflaa sequence via PCR site-directed mutation using primers: F: gacaaaagccttcctactacact; R: tagtaggaagggcttttgtctatttgagtaa tctgctgtatcctg. Embryos at the one-cell stage were transfected with the WT kif1aa-Tol2 or A433D kif1aa-Tol2 plasmid with transposase via cytoplasmic microinjection.

\section{Behavior Monitoring and Local Field Potential Recording}

For behavior monitoring, zebrafish larvae at 5 days postfertilization (d.p.f.) were placed in 24-well Falcon culture dishes that contained embryo medium. A charge-coupled device (CCD) camera and EthoVision 3.1 locomotion tracking software were used to monitor the swim activity of the mutant (A433D kiflaa-Tol2) and WT (WT kiflaa-Tol2) larvae, and each larva was monitored for $15 \mathrm{~min}$. The swim activity was categorized by three observers who were blind to the larva phenotype as stage 0 (baseline activity), stage 1 (small increase in swim activity), and stage 2 (large increase in movement) (Hortopan et al., 2010).

For local field potential recording, zebrafish larvae at 5 d.p.f. were immobilized in low-melting temperature agarose. A glass filled with $2 \mathrm{M} \mathrm{NaCl}$ was placed in the optic tecum of zebrafish larvae, and each recording was performed for $15 \mathrm{~min}$ in the current clamp mode with high-pass filtering above $0.1 \mathrm{~Hz}$ and low-pass filtering below $1 \mathrm{kHz}$. The digital gain was 10 [MultiClamp 700B Amplifier (Axon, Sunnyvale, CA, USA) and Digidata1440A (Axon, Sunnyvale, CA, USA)]. Spontaneous events were defined as instances in which the amplitude exceeded three times the background noise (Schubert et al., 2014). Clampfit 10.3 (Molecular Devices, Sunnyvale, CA, USA) software was used for the data analyses.

\section{Statistical Analysis}

The measurement data (means \pm SDs) from two groups were compared using Student's $t$ test, and the data from more than two parameters were analyzed by two-way ANOVA. Chi-square tests were used to compare the differences in the behavior and local field potential (LFP) of larvae. $P<0.05$ was considered statistically significant, and the statistical analyses were performed using GraphPad Prism version 7.0 (GraphPad Software).

\section{RESULTS}

\section{Clinical Characteristics of Patients in the Family}

The family of interest had six affected patients over three generations, which suggests autosomal dominant inheritance (Figure 1A). The clinical characteristics of the epilepsy patients in the family are described in Table 1. All the affected individuals presented with generalized tonic-clonic seizures without mental retardation, and three of these patients suffered from diabetes mellitus. The seizure onset age of patient III2 was 12 years, and electroencephalogram (EEG) showed generalized sharp waves that were prevalent bilaterally (Figure 1B). She started treatment with levetiracetam, which resulted in good control of her seizures. The seizure onset ages of the other patients, namely, 
A

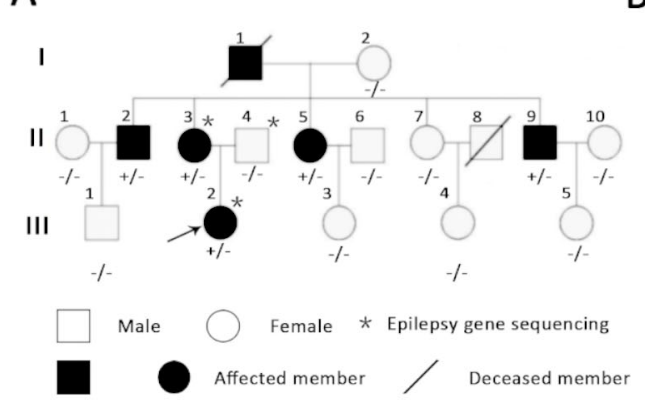

C
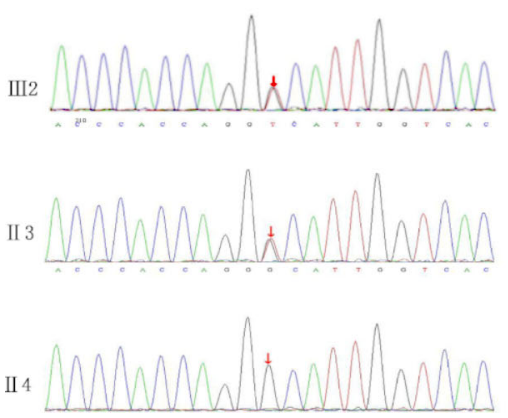

D

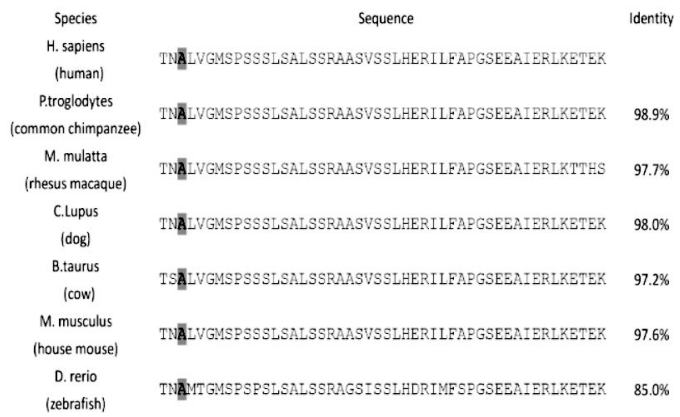

FIGURE 1 | Rare variants in KIF1A detected in a family and KIF1A are conserved across species. (A) Rare mutation in KIF1A detected in a family. (B) Electroencephalogram (EEG) of the proband (III2) showing generalized sharp waves. (C) The analysis of sequences of affected girl (III2), affected mother (II3), and unaffected father (II4). (D) The amino acids coded by variation are conserved across species as showing in the boxed. The last column shows identity with the human protein. The gray boxes highlight the amino acids mentioned in the manuscript.

II2, II3, II5, and II9, were 18, 16, 21, and 24 years, respectively. None of these individuals had received standard anti-epileptic therapy. Patient II9 presented an abnormal brain CT scan due to ischemic stroke, and the CT scans of the brains of the other patients were normal.

We first performed customized sequencing of epilepsy-related genes of two epilepsy patients (III2 and II3) and one unaffected individual (II4) and identified a possible causal variant (c.1190C $>$ A, p. Ala397Asp) on the neck-coiled coil of KIF1A in heterozygosis. The frequency of this mutation in the Genome Aggregation Database (http://gnomad.broadinstitute.org) is 0.000008133 . Sanger sequencing of this gene in all members of this family was then performed to assess the segregation of KIF1A mutations. This rare mutation was found in all affected family members and was not detected in the unaffected family
B

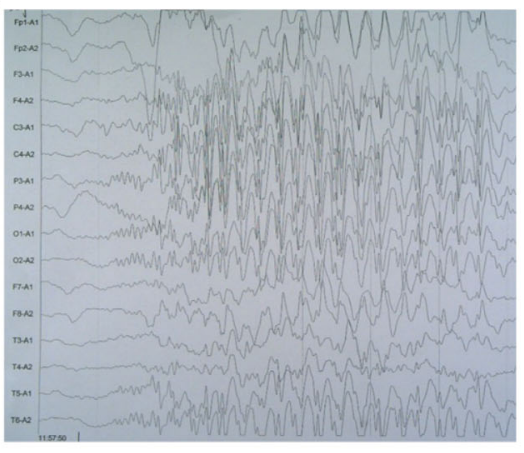
. . 


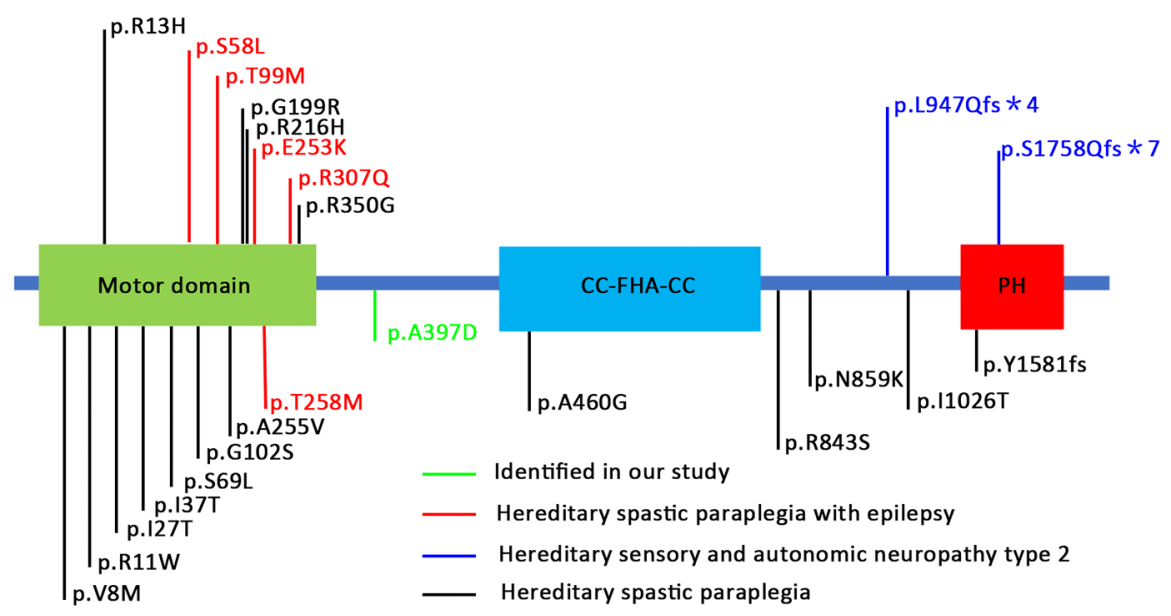

FIGURE 2 | Schematic representation of KIF1A protein and locations of mutations in human KIF1A associated with various neuronal disorders. These mutations are distributed in various locations and domains of the KIF1A subunit protein peptide (Niwa et al., 2016; Iqbal et al., 2017; Riviere et al., 2011; Chiba et al., 2019).

primary cultured neurons (Supplementary Figures S1A, B). Also, we revealed the wild type protein and mutant protein expressed equally in soma and nerites (Supplementary Figures S1C, D).

In general, increased neuronal firing is due to intrinsic excitability or altered synaptic transmission. Therefore, we tested the intrinsic excitability of the neurons through whole-cell patchclamp recordings of the primary cultured neurons. The primary cultured neurons were transfected with the indicated plasmid at
DIV7, and the whole-cell recording was performed at DIV14. We first checked the resting membrane potential of each neuron and found no difference between the WT and mutant groups (Figure 3C). A depolarizing current of $500 \mathrm{~ms}$ was then applied in the current clamp mode starting from $-30 \mathrm{pA}$ and at increments of 10 $\mathrm{pA}$ to induce neuronal firming (Figure 3A). We analyzed the injected current intensity between the two groups and found no difference (Figure 3D). The action potential recordings revealed

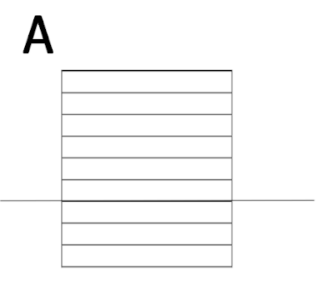

currents were injected from -30pA to $60 \mathrm{pA}$ (step by $10 \mathrm{pA}$ ) lasting for $500 \mathrm{~ms}$ C

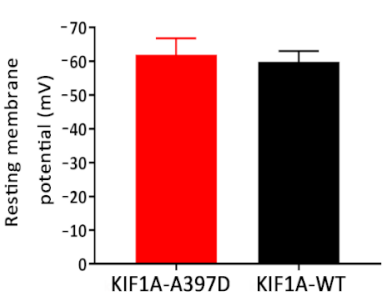

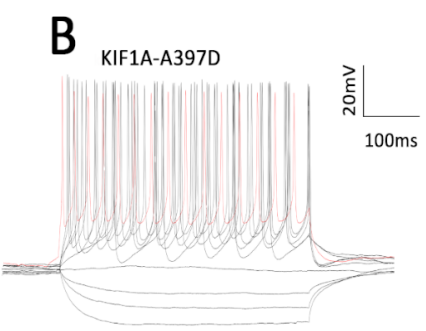
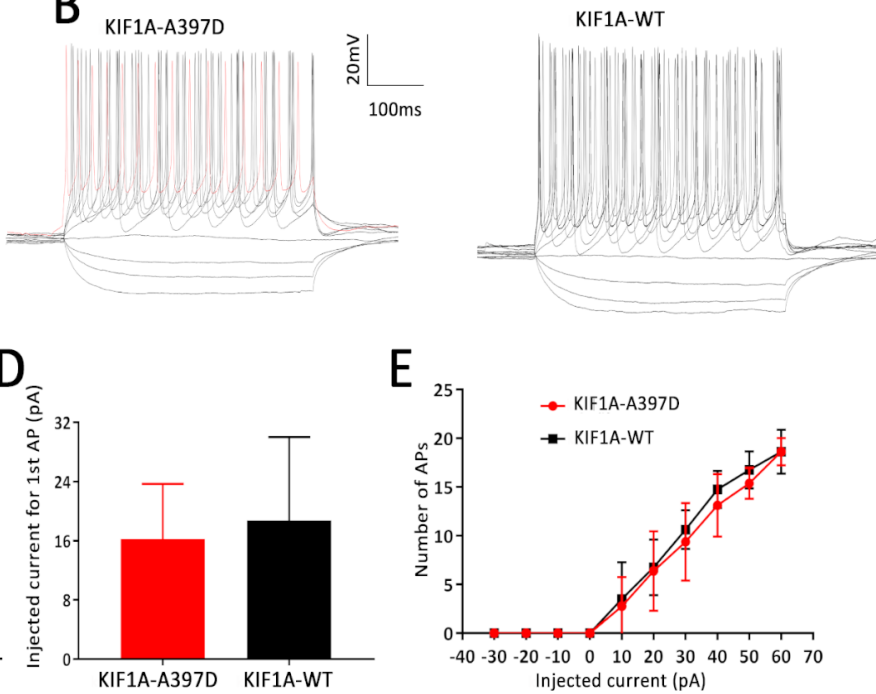

FIGURE 3 | Effect of mutant KIF1A on the intrinsic excitability of primary cultured neurons. (A) Recording paradigm of passive excitability in the excitatory cultured neurons. (B) Examples of the AP responses to superimposed current steps recorded from primary cultured GFP-positive hippocampal neurons transfected with the mutant or wild-type KIF1A plasmid. (C) Resting membrane potential of the examined neurons from two groups ( $\mathrm{n}=8 \mathrm{neurons}$ in each group, Student's $t$ test). (D) Injected currents used to induce the first spikes ( $n=8$ neurons in each group, Student's $t$ test). (E) Number of APs induced by the injected currents in the primary cultured GFP-positive hippocampal neurons transfected with the mutant or wild-type KIF1A plasmid ( $\mathrm{n}=8$ neurons in each group, two-way ANOVA). 
that the number of action potentials induced by the injected currents was unaffected (Figures 3B, E). In conclusion, mutant KIF1A did not affect the intrinsic excitability of neurons.

\section{Effect of Mutant KIF1A on Miniature Excitatory Post-Synaptic Currents and Miniature Inhibitory Post-Synaptic Currents in Primary Cultured Neurons}

We did not find any difference in intrinsic excitability between the WT and mutant groups and therefore speculate that mutant KIF1A might disrupt synaptic transmission. To investigate this hypothesis, we obtained whole-cell patch-clamp recordings of mEPSCs and mIPSCs in neurons at DIV14 that had been transfected with the plasmid at DIV7. Compared with the expression of WT KIF1A, the expression of mutant KIF1A resulted in a significant increase in the frequency of mEPSCs in neurons, whereas the amplitude of mEPSCs did not show a significantly difference between neurons expressing WT and those expressing mutant KIF1A (Figures 4A-C). The mIPSC analysis revealed that the frequency and amplitude did not differ between the two neuron groups (Figures 4D-F). Taken together, these results indicate that the expression of mutant KIF1A leads to an enhanced excitatory synaptic transmission.

\section{Effect of Mutant KIF1A on Neuronal Development}

Previous studies have shown that KIF1A is a neuron-specific expressed protein (Okada et al., 1995) and have demonstrated that knockout of KIF1A in mice results in death within $24 \mathrm{~h}$ after birth (Yonekawa et al., 1998). We thus hypothesize that KIF1A plays an important role in neuronal development and that mutant KIF1A might be involved in neuronal development. To test this hypothesis, we transfected primary cultured neurons with a plasmid encoding either WT or mutant KIF1A at DIV7 and fixed these cells at DIV10. The resulting neuronal branching was analyzed through Sholl analysis, and surprisingly, no difference in neuronal branching was found between the WT and mutant neurons (Figures 5A, B). We subsequently measured the number of primary and secondary neurites and found that mutant KIF1A did not affect the number of neurites (Figures 5C, D). In brief, mutant KIF1A does not affect neuronal development.

\section{Effect of Mutant KIF1A on Excitatory Synapses}

KIF1A is essential for synaptogenesis in the hippocampus (Kondo et al., 2012). In our study, the expression of mutant KIF1A resulted in a significant increase in the frequency of mEPSCs in neurons compared with that observed with WT KIF1A. The increased frequency of mEPSCs might be due to increased presynaptic vesicle release probability, and the increased number of excitatory synapses per neuron is also responsible for the observed increase in the frequency of mEPSCs. Dendritic spines contain the majority of the excitatory synapses of hippocampal pyramidal neurons, and changes in the spine density lead to alterations in the mEPSC frequency and are associated with aberrations in synaptic plasticity. To determine whether the mutant KIF1A affects the dendritic spines, we transfected neurons with a plasmid construct encoding the WT or mutant KIF1A at DIV7 and fixed these neurons at DIV16. Consistent with our hypothesis, the neurons transfected with mutant KIF1A exhibited a
A

KIF1A-A397D

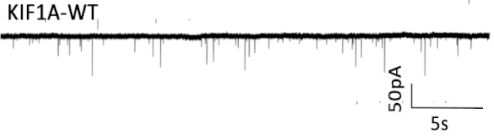

D

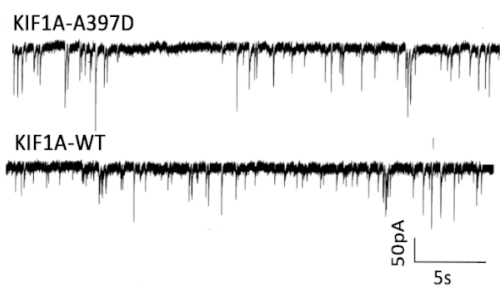

B

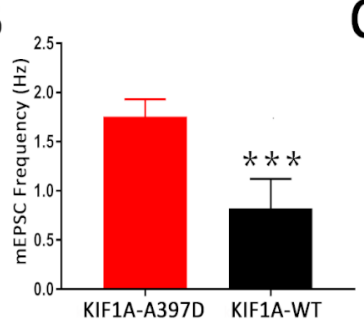

C

E

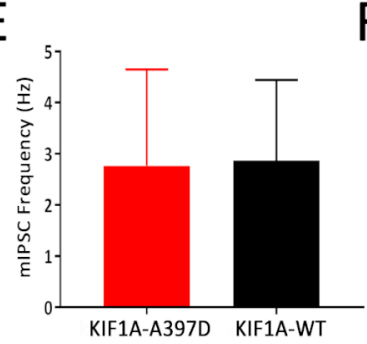

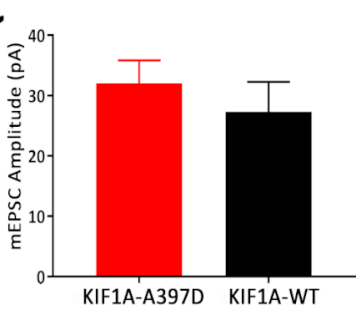

$\mathrm{F}$

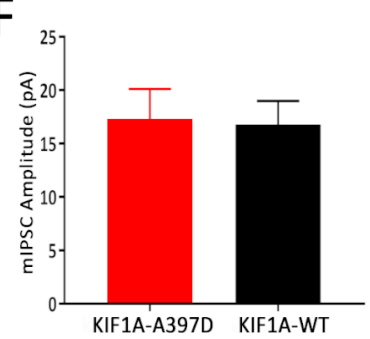

FIGURE 4 | Effect of mutant KIF1A on the miniature excitatory post-synaptic currents (mEPSCs) and miniature inhibitory post-synaptic currents (mIPSCs) of primary cultured neurons. (A) Representative trace of mEPSCs from cultured hippocampal neurons transfected with the mutant or wild-type KIF1A plasmid at a holding potential of $-70 \mathrm{mV}$. (B, C) Bar graph analysis of the mEPSC frequency and amplitude ( $\mathrm{n}=8$ neurons in each group, ${ }^{\star \star \star} p<0.001$, Student's $t$ test). (D) Representative trace of mIPSCs from cultured hippocampal neurons transfected with the mutant or wild-type KIF1A plasmids at a holding potential of -70 mV. (E, F) Bar graph analysis of the mIPSC frequency and amplitude [ $=10$ in the mutant group and 8 in the wild type (WT) group, Student's $t$ test]. 


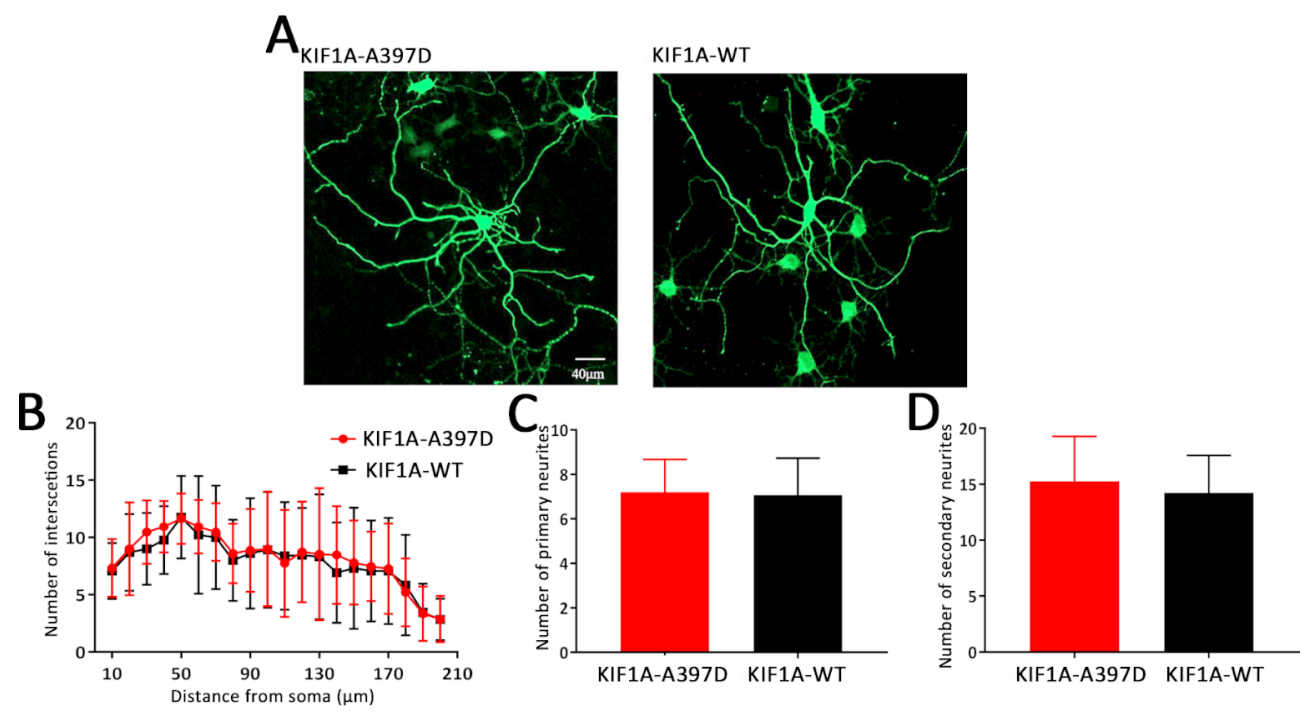

FIGURE 5 | Effect of mutant KIF1A on neuronal development. (A) Representative image of cultured primary hippocampal neurons obtained through a Sholl analysis. The radius interval between circles was $10 \mu \mathrm{m}$ per step and ranged from 10 to $210 \mu \mathrm{m}$ from the center of the neuronal soma. (B) Sholl analysis of neurons expressing the mutant ( $n=15$ neurons) or wild-type protein ( $n=13$ neurons) (two-way ANOVA). (C, D) Number of primary and secondary neurites of neurons expressing the mutant ( $n=15$ neurons) or wild-type protein ( $n=13$ neurons) (Student's $t$ test).

significantly higher density of dendritic spines and vGLUT compared with the neurons transfected with WT KIF1A (Figures 6A-D). To further verify the excitatory synapse formation, vGLUT-positive and PSD-95 clusters were examined using double immunofluorescence staining (Figures $6 \mathrm{E}, \mathrm{F}$ ), our results suggest that mutant KIF1A is responsible for the observed increase in the excitatory synaptic density.

\section{Effect of Mutant kif1aa on the Behavior of and Local Field Potentials in Zebrafish}

We subsequently investigated the functional consequence of mutant kif1a in vivo by establishing a zebrafish model. Zebrafish have two kifla homologue genes termed kiflaa and $k i f 1 a b$ that encode two protein isoforms, Kiflaa and Kiflab, which show 85 and $80 \%$ identity to human KIF1A, respectively. The mutant alanine in the affected patients corresponds to A433 in Kiflaa and A410 in Kiflab. Both of these genes have the same function in neurons, and kiflaa, which is also located on chromosome 2, and Kiflaa share higher (85\%) similarity with human KIF1A. Therefore, we selected zebrafish kif1aa for our zebrafish experiment.

To establish the zebrafish model, we cloned mutant and WT kiflaa into the tol2 expression vector (WT kif1aa-Tol2 or A433D kif1aa-Tol2) and evaluated the expression of Kiflaa in zebrafish larvae using GFP. Mutant (A433D kif1aa-Tol2) or WT kif1aa (WT kif1aa-Tol2) was introduced into embryos at the one-cell stage by cytoplasmic microinjection (Figure 7A). Three days later, neither WT nor mutant zebrafish exhibited gross dysmorphologies (Figure 7B). Normal-looking zebrafish larvae displaying a touch response at 5 d.p.f. were selected for behavior monitoring. These zebrafish larvae were placed in a well of a 24well falcon plate, and the motors of the freely swimming fish were observed using a stereomicroscope. The results showed that $67.2 \%$ of the WT larvae displayed normal behavior that could be characterized as S0 (baseline activity), and only $11.5 \%$ of the WT larvae exhibited abnormal behaviors that could be characterized S2 (large increase in movement). The analysis of the mutant larvae revealed that $40.6 \%$ displayed excessive motor activity that could be characterized as S2, and the percentage of larvae showing baseline activity (S0) was $39.0 \%$, which was significantly lower than that found for the WT larvae (Figure 7C). To determine whether the missense mutation in Kiflaa resulted in excessive brain electrophysical activity, we obtained local field potential recordings from the zebrafish tectum at 5 d.p.f. and observed spontaneous epileptiform activity (polyspike discharges) in $42.2 \%$ of mutant larvae and $9.8 \%$ of WT larvae (this difference was significant) (Figures 7D, E).

\section{DISCUSSION}

Although it has been confirmed that most patients with epilepsy have genetic epilepsy, the transmission of the epilepsy phenotype is clearly autosomal dominant or autosomal recessive. However, the genetic etiology of generalized epilepsy in the majority of patients is unknown. In recent decades, extensive use of highthroughput sequencing in recent years has confirmed that a large amount of gene alterations might be responsible for epileptogenesis (Helbig et al., 2016; McTague et al., 2016). In our study, we identified a rare mutation of KIF1A in a family with six affected patients over three generations using a panel of epilepsy-related genes and Sanger sequencing. We first investigated the possible mechanism in primary cultured neurons and found that mutant KIF1A increased the density of 
A

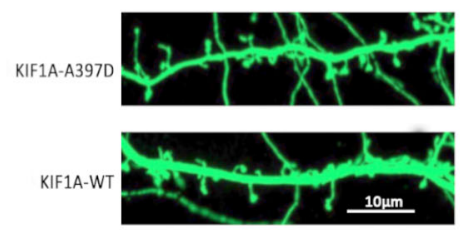

C

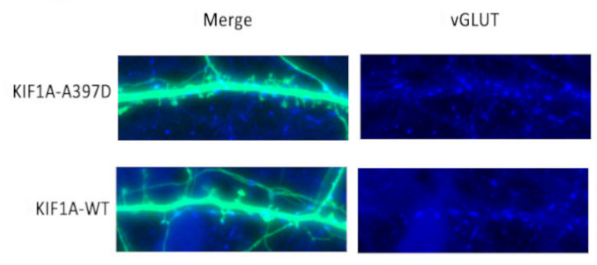

$E$

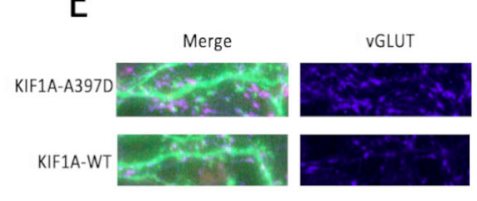

B
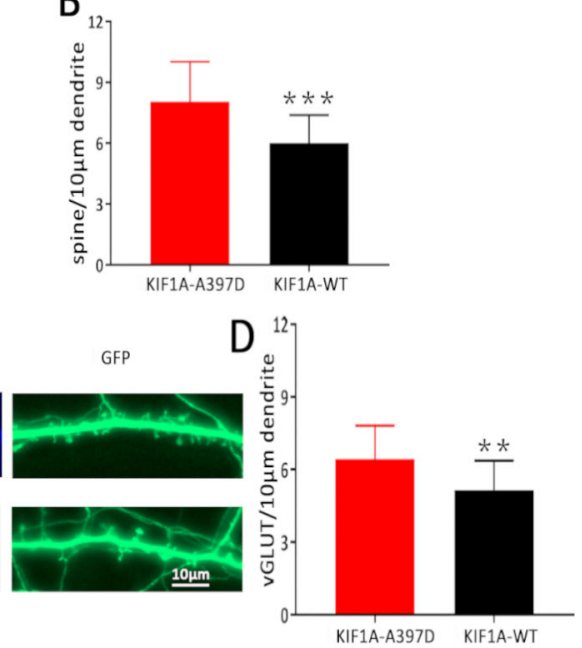

KIF1A-A397D KIF1A-WT

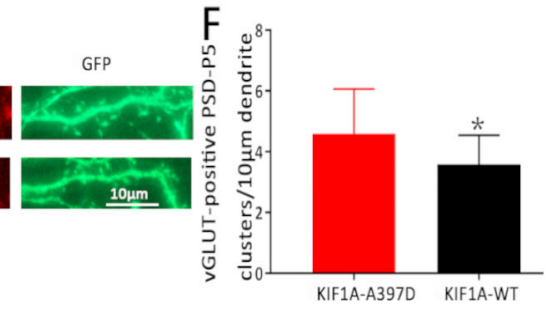

FIGURE 6 | Effect of mutant KIF1A on excitatory synapses. (A) Representative image of dendritic spines at DIV16 from neurons transfected with the mutant or wildtype KIF1A plasmid. (B) Total spines/10 $\mu$ m of neurons expressing the mutant ( $n=26$ neurons) or wild-type protein ( $n=23$ neurons). ( ${ }^{\star \star \star} p<0.001$, Student's $t$ test). (C) Representative image of VGLUT at DIV16 from neurons transfected with the mutant or wild-type KIF1A plasmid. (D) Total vGLUT/10 $\mu$ m of neurons expressing the mutant ( $n=24$ neurons) or wild-type protein ( $n=21$ neurons). ( ${ }^{\star *} p<0.01$, Student's $t$ test). (E) Representative image of excitatory synapses (vGLUT-positive PSD-95 clusters) at DIV16 from neurons transfected with the mutant or wild-type KIF1A plasmid. (F) Total excitatory synapses (vGLUT-positive PSD-95 clusters)/10 $\mu \mathrm{m}$ of neurons expressing the mutant ( $\mathrm{n}=19$ neurons) or wild-type protein ( $\mathrm{n}=18$ neurons). $\left({ }^{*} p<0.05\right.$, Student's $t$ test).

excitatory synapse, which indicates that the mutation might be a gain-of-function mutation. A functional test using zebrafish showed that the mutation resulted in epileptic activity.

KIF1A, a kinesin-3 member, is a unique monomeric neuronspecific motor protein. is mainly involved in the anterograde transport of synaptic vesicle proteins in axons, such as synaptotagmin, synaptophysin, and Rab3A (Okada et al., 1995). Several studies have shown that KIF1A is also responsible for neuron migration and synaptic plasticity (McVicker et al., 2016; Stucchi et al., 2018). KIF1A has four sections: a motor domain, a neck coiled-coil region, a CC-FHA-CC domain, and a globular tail/ pleckstrin homology domain (Huo et al., 2012; Lee et al., 2015). The motor domain mainly includes a nucleotide catalytic binding site and a microtubule-binding site. The neck coiled-coil region, which is attached to the motor domain, is very flexible, undergoes different conformational changes at different nucleotide-binding states, and produces tension through docking and separation from the motor domain. The CC-FHA-CC domain is located in the middle of the isoform. The end of the stalk region contains the globular tail/ pleckstrin homology domain, which recognizes vesicles and membranous organelles (Hirokawa et al., 2009) Thus far, limited studies have investigated the role of KIF1A in the origin of epilepsy. Previous studies have reported that several patients with mutations in KIF1A present recurrent seizures; however, these studies investigated only the correlation of neuropathy and brain malformation with mutations in KIF1A (Esmaeeli Nieh et al., 2015; Lee et al., 2015; Hotchkiss et al., 2016; Megahed et al., 2016; Cheon et al., 2017; Demily et al., 2018). All of these reported mutations accompanied by recurrent seizures were located in the motor domain of KIF1A and resulted in decreased motor activity. In our study, we found a missense mutation located in the neck coiled-coil region, and the maintenance of epileptic susceptibility observed with this mutant KIF1A was mainly an outcome of enhanced excitatory synaptic plasticity rather than individual properties. We examined the mEPSCs and mIPSCs of primary cultured neurons and found that only the frequency of mEPSCs was altered in the mutant group, which indicates effects on synaptogenesis.

To confirm this finding, we investigated synaptogenesis in neurons. Dendritic spines are the synaptic component in the majority of excitatory neurons, and the observed increase in spine density and vGLUT is consistent with the increased frequency of mEPSCs. To some extent, spine alterations signify the neuronal network dynamics (Cooke and Woolley, 2009). Previous studies have demonstrated that aberrant alterations in the dendritic spine density are commonly observed in brain 

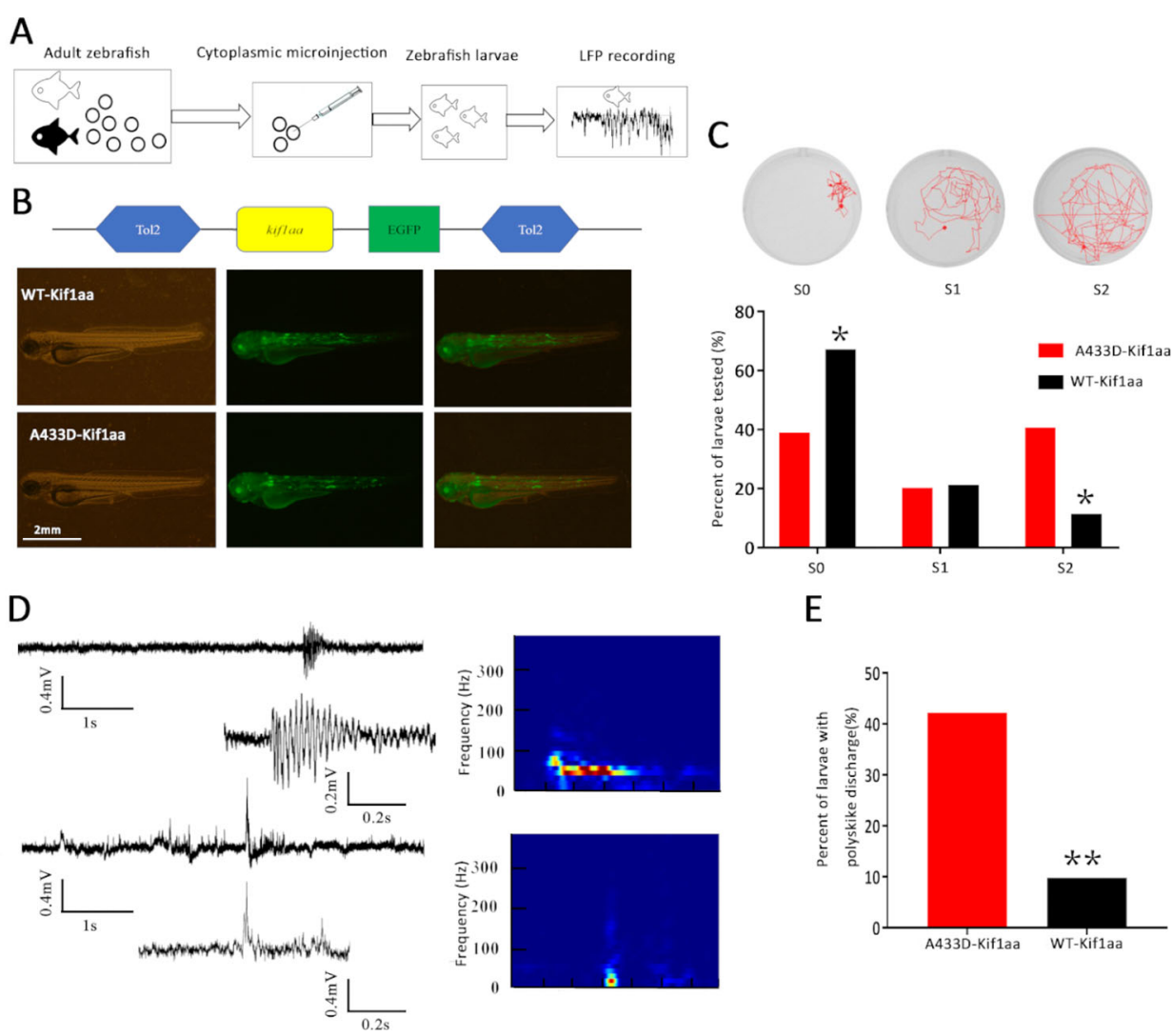

$\mathrm{E}$

FIGURE 7 | Overexpression of mutant Kif1aa causes abnormal behavior and epileptiform discharges in transgenic zebrafish larvae. (A) Graphic representation of the experimental protocol used for the zebrafish studies. (B) Representative images of zebrafish larvae microinjected with A433D-kif1aa ( $n=64)$ or wild type (WT)-kif1aa ( $n=61)$ selected for local field recordings. The distribution of the labeled protein is shown by green fluorescence. (C) Seizure behaviors of zebrafish larvae. The sample locomotion tracking plots are shown on the top (SO, baseline activity; S1, small increase in swim activity; and S2, large increase in movement. The bar plot shows the percentage of tested larvae recorded during locomotion tracking ( ${ }^{*} p<0.05, \chi^{2}$ test). (D) Representative trace of spontaneous epileptiform activity recorded from zebrafish larvae. The top trace represents a typical epileptiform pattern observed in gap-free recordings. The bottom trace shows a high-resolution magnification of the selected epileptiform events, and the frequency spectrum corresponding to the selected local field potentials (LFPs) is shown on the right. Color scale: blue (low magnitude) to red (high magnitude). (E) Percentage of larvae with abnormal epileptiform activity after overexpression of A433D ( $n=64)$ or WT Kif1aa $(n=61) .\left({ }^{* *} p<0.01, \chi^{2}\right.$ test $)$.

samples from epilepsy patients and epilepsy animal models (Jiang et al., 1998; Wong and Guo, 2013). Dendritic spine abnormalities might increase the hyperexcitable circuits or intrinsic properties of neurons that might cause seizures, and seizures also result in damage to dendrites and dendritic spines, which might contribute to progressive recurrent seizures, mental disorders, memory disturbances, and other neurological deficits in epilepsy patients (Jimenez-Mateos et al., 2015; Awad et al., 2016; Carter et al., 2017)

Previous study suggest that KIF1A mutations that cause hereditary spastic paraplegia are loss-of-function mutations that decrease motility (Esmaeeli Nieh et al., 2015). However, not all mutations are loss-of-function. Recently, Chiba et al. revealed gain-of-function mutations in KIF1A, V8M, A255V, and R350G that cause overactivation of KIF1A motor activity in vitro. KIF1A (V8M), KIF1A (A255V), and KIF1A (R350G) increased the landing rate (20-fold or 10 -fold higher than the WT), and the velocity of KIF1A (V8M) and KIF1A (R350G) was
2- and 3-fold faster, respectively, than WT KIF1A (Chiba et al., 2019). Other mutations (E412K, G598R, and E612K) showed with similar phenomena (Niwa et al., 2016). However, the molecular mechanism of these changes remains unclear, and the authors speculate that these mutations could conceivably alter the motor enzymatic rate or lead to varying degrees of autoinhibition release. Interestingly, the abovementioned gainof-function mutation results in a reduced synaptic size in Caenorhabditis elegans. In clinical presentation, the phenotype of gain-of-function mutant individuals was milder than in lossof-function mutant individuals (Klebe et al., 2012; Chiba et al., 2019). The gain-of-function mutations located in the motor domain increased the landing rate or velocity. In our study, the KIF1A (A397D) mutation, which is located in the neck coiledcoil (mapped between the motor domain and CC-FHA-CC domain), was also revealed as a gain-of-function mutation (increased excitatory synapse). Therefore, we speculate that the KIF1A (A397D) mutant may have caused increased excitatory 
synaptic density and seizure activity by increasing the landing rate and/or velocity, but these increases are not sufficient for triggering other neuropathies [e.g., spastic paraplegia (SPG) and intellectual disability].

To validate that mutant KIF1A is a gain-of-function mutation in vivo, we constructed overexpression transgenic zebrafish using the tol 2 vector. Zebrafish have two kifla homologous genes, kiflaa and kiflab. The kiflaa gene is located on chromosome 2, and its encoded protein, Kiflaa, shares $85 \%$ identity with human KIF1A. Therefore, we selected the corresponding residue A433D in zebrafish Kif1aa for the zebrafish experiments. Interestingly, our functional experiment demonstrated that the overexpression of Kiflaa in the vertebrate in vivo system results in abnormal seizure-like behavior and epileptiform-like discharges.

In conclusion, our data provide evidence demonstrating that the kinesin superfamily member KIF1A is involved in epileptogenesis. The identification of other epilepsy patients with mutations in this gene should further confirm the role of KIF1A and might provide further insights into the full clinical spectrum. The functional-level results indicated that the mutation in the neck linker of KIF1A resulted in increased dendritic spines, which might be a main cause of aberrant neuronal circuits in the brain.

\section{DATA AVAILABILITY STATEMENT}

The datasets generated for this study can be found in NCBI GenBank accession MN897723.

\section{ETHICS STATEMENT}

The studies involving human participants were reviewed and approved by Ethics Committee of Chongqing Medical University. The patients/participants provided their written informed consent to participate in this study. The animal study was reviewed and approved by Ethics Committee of Chongqing Medical University. Written informed consent was obtained from the owners for the participation of their animals in this

\section{REFERENCES}

Awad, P. N., Sanon, N. T., Chattopadhyaya, B., Carrico, J. N., Ouardouz, M., Gagne, J., et al. (2016). Reducing premature KCC2 expression rescues seizure susceptibility and spine morphology in atypical febrile seizures. Neurobiol. Dis. 91, 10-20. doi: 10.1016/j.nbd.2016.02.014

Berg, A. T., Berkovic, S. F., Brodie, M. J., Buchhalter, J., Cross, J. H., van Emde Boas, W., et al. (2010). Revised terminology and concepts for organization of seizures and epilepsies: report of the ILAE commission on classification and terminology 2005-2009. Epilepsia 51 (4), 676-685. doi: 10.1111/j.15281167.2010.02522.x

Carter, A. N., Born, H. A., Levine, A. T., Dao, A. T., Zhao, A. J., Lee, W. L., et al. (2017). Wortmannin attenuates seizure-induced Hyperactive PI3K/Akt/ mTOR signaling, impaired memory, and spine dysmorphology in rats. eNeuro 4 (3), 1-15. doi: 10.1523/ENEURO.0354-16.2017

Cheon, C. K., Lim, S. H., Kim, Y. M., Kim, D., Lee, N. Y., Yoon, T. S., et al. (2017). Autosomal dominant transmission of complicated hereditary spastic paraplegia due to a dominant negative mutation of KIF1A, SPG30 gene. Sci. Rep. 7 (1), 12527-12536. doi: 10.1038/s41598-017-12999-9 study. Written informed consent was obtained from the individual(s) for the publication of any potentially identifiable images or data included in this article

\section{AUTHOR CONTRIBUTIONS}

YG, YM, XW, and XT conceived the project and designed the experiments. YG, YC, MY, XX, ZL, JM, HC, YH, YM, and XT performed the experiments. YG, XW, and XT wrote the manuscript. All authors revised and approved the final version of the manuscript.

\section{FUNDING}

This work was supported by grants from the National Natural Science Foundation of China (81671301, 81901332, and 81701279), Chongqing Nature Science Foundation Project (csts2019jcyj-msxmX0184) and Cultivating Fund of the First Affiliated Hospital of Chongqing Medical University (PYJJ2019201 and PYJJ2018-11).

\section{ACKNOWLEDGMENTS}

We are thankful for the members of the family for their participation and help in this study. We thank Koichi Kawakami (National Institute of Genetics, Japan) for providing Tol2 expression vector (pT2AL200R150G) and transposase plasmid (pCS-zT2TP).

\section{SUPPLEMENTARY MATERIAL}

The Supplementary Material for this article can be found online at: https://www.frontiersin.org/articles/10.3389/fgene.2020.00061/ full\#supplementary-material

Chiba, K., Takahashi, H., Chen, M., Obinata, H., Arai, S., Hashimoto, K., et al (2019). Disease-associated mutations hyperactivate KIF1A motility and anterograde axonal transport of synaptic vesicle precursors. Proc. Natl. Acad. Sci. U. S. A. 116 (37), 18429-18434. doi: 10.1073/pnas.1905690116

Citterio, A., Arnoldi, A., Panzeri, E., Merlini, L., D'Angelo, M. G., Musumeci, O., et al. (2015). Variants in KIF1A gene in dominant and sporadic forms of hereditary spastic paraparesis. J. Neurol. 262 (12), 2684-2690. doi: 10.1007/ s00415-015-7899-9

Cooke, B. M., and Woolley, C. S. (2009). Effects of prepubertal gonadectomy on a male-typical behavior and excitatory synaptic transmission in the amygdala. Dev. Neurobiol. 69 (2-3), 141-152. doi: 10.1002/dneu.20688

Demily, C., Lesca, G., Poisson, A., Till, M., Barcia, G., Chatron, N., et al. (2018). Additive effect of variably penetrant 22q11.2 duplication and pathogenic mutations in autism spectrum disorder: to which extent does the tree hide the forest? J. Autism Dev. Disord. 48 (8), 2886-2889. doi: 10.1007/s10803-0183552-7

Esmaeeli Nieh, S., Madou, M. R., Sirajuddin, M., Fregeau, B., McKnight, D., Lexa, K., et al. (2015). De novo mutations in KIF1A cause progressive encephalopathy and brain atrophy. Ann. Clin. Transl. Neurol. 2 (6), 623-635. doi: 10.1002/acn3.198 
Helbig, I., Heinzen, E. L., and Mefford, H. C. (2016). Primer Part 1-The building blocks of epilepsy genetics. Epilepsia 57 (6), 861-868. doi: 10.1111/epi.13381

Hirokawa, N., Nitta, R., and Okada, Y. (2009). The mechanisms of kinesin motor motility: lessons from the monomeric motor KIF1A. Nat. Rev. Mol. Cell Biol. 10 (12), 877-884. doi: 10.1038/nrm2807

Hortopan, G. A., Dinday, M. T., and Baraban, S. C. (2010). Spontaneous seizures and altered gene expression in GABA signaling pathways in a mind bomb mutant zebrafish. J. Neurosci. 30 (41), 13718-13728. doi: 10.1523/ JNEUROSCI.1887-10.2010

Hotchkiss, L., Donkervoort, S., Leach, M. E., Mohassel, P., Bharucha-Goebel, D. X., Bradley, N., et al. (2016). Novel de novo mutations in KIF1A as a cause of hereditary spastic paraplegia with progressive central nervous system involvement. J. Child Neurol. 31 (9), 1114-1119. doi: 10.1177/0883073816639718

Huo, L., Yue, Y., Ren, J., Yu, J., Liu, J., Yu, Y., et al. (2012). The CC1-FHA tandem as a central hub for controlling the dimerization and activation of kinesin-3 KIF1A. Structure 20 (9), 1550-1561. doi: 10.1016/j.str.2012.07.002

Iqbal, Z., Rydning, S. L., Wedding, I. M., Koht, J., Pihlstrom, L., Rengmark, A. H., et al. (2017). Targeted high throughput sequencing in hereditary ataxia and spastic paraplegia. PloS One 12 (3), e0174667. doi: 10.1371/journal.pone.0174667

Jiang, M., Lee, C. L., Smith, K. L., and Swann, J. W. (1998). Spine loss and other persistent alterations of hippocampal pyramidal cell dendrites in a model of early-onset epilepsy. J. Neurosci. 18 (20), 8356-8368. doi: 10.1523/ JNEUROSCI.18-20-08356.1998

Jimenez-Mateos, E. M., Engel, T., Merino-Serrais, P., Fernaud-Espinosa, I., Rodriguez-Alvarez, N., Reynolds, J., et al. (2015). Antagomirs targeting microRNA-134 increase hippocampal pyramidal neuron spine volume in vivo and protect against pilocarpine-induced status epilepticus. Brain Struct. Funct. 220 (4), 2387-2399. doi: 10.1007/s00429-014-0798-5

Klebe, S., Lossos, A., Azzedine, H., Mundwiller, E., Sheffer, R., Gaussen, M., et al. (2012). KIF1A missense mutations in SPG30, an autosomal recessive spastic paraplegia: distinct phenotypes according to the nature of the mutations. Eur. J. Hum. Genet. 20 (6), 645-649. doi: 10.1038/ejhg.2011.261

Kondo, M., Takei, Y., and Hirokawa, N. (2012). Motor protein KIF1A is essential for hippocampal synaptogenesis and learning enhancement in an enriched environment. Neuron 73 (4), 743-757. doi: 10.1016/j.neuron.2011.12.020

Lee, J. R., Srour, M., Kim, D., Hamdan, F. F., Lim, S. H., Brunel-Guitton, C., et al. (2015). De novo mutations in the motor domain of KIF1A cause cognitive impairment, spastic paraparesis, axonal neuropathy, and cerebellar atrophy. Hum. Mutat. 36 (1), 69-78. doi: 10.1002/humu.22709

McTague, A., Howell, K. B., Cross, J. H., Kurian, M. A., and Scheffer, I. E. (2016). The genetic landscape of the epileptic encephalopathies of infancy and childhood. Lancet Neurol. 15 (3), 304-316. doi: 10.1016/S1474-4422(15)00250-1

McVicker, D. P., Awe, A. M., Richters, K. E., Wilson, R. L., Cowdrey, D. A., Hu, X., et al. (2016). Transport of a kinesin-cargo pair along microtubules into dendritic spines undergoing synaptic plasticity. Nat. Commun. 7, 12741. doi: $10.1038 /$ ncomms 12741

Megahed, H., Nicouleau, M., Barcia, G., Medina-Cano, D., Siquier-Pernet, K., Bole-Feysot, C., et al. (2016). Utility of whole exome sequencing for the early diagnosis of pediatric-onset cerebellar atrophy associated with developmental delay in an inbred population. Orphanet J. Rare Dis. 11 (1), 57. doi: 10.1186/ s13023-016-0436-9

Miller, L. L., Pellock, J. M., DeLorenzo, R. J., Meyer, J. M., and Corey, L. A. (1998). Univariate genetic analyses of epilepsy and seizures in a population-based twin study: the virginia twin registry. Genet. Epidemiol. 15 (1), 33-49. doi: 10.1002/ (SICI) 1098-2272(1998) 15:1<33::AID-GEPI3>3.0.CO;2-5

Niwa, S., Lipton, D. M., Morikawa, M., Zhao, C., Hirokawa, N., Lu, H., et al. (2016). Autoinhibition of a Neuronal Kinesin UNC-104/KIF1A regulates the size and density of synapses. Cell Rep. 16 (8), 2129-2141. doi: 10.1016/ j.celrep.2016.07.043

Okada, Y., Yamazaki, H., Sekine-Aizawa, Y., and Hirokawa, N. (1995). The neuron-specific kinesin superfamily protein KIF1A is a unique monomeric motor for anterograde axonal transport of synaptic vesicle precursors. Cell 81 (5), 769-780. doi: 10.1016/0092-8674(95)90538-3

Riviere, J. B., Ramalingam, S., Lavastre, V., Shekarabi, M., Holbert, S., Lafontaine, J., et al. (2011). KIF1A, an axonal transporter of synaptic vesicles, is mutated in hereditary sensory and autonomic neuropathy type 2. Am. J. Hum. Genet. 89 (2), 219-230. doi: 10.1016/j.ajhg.2011.06.013

Scheffer, I. E., French, J., Hirsch, E., Jain, S., Mathern, G. W., Moshe, S. L., et al. (2016). Classification of the epilepsies: New concepts for discussion and debate-Special report of the ILAE classification task force of the commission for classification and terminology. Epilepsia Open 1 (1-2), 37-44. doi: 10.1002/ epi4.5

Schubert, J., Siekierska, A., Langlois, M., May, P., Huneau, C., Becker, F., et al. (2014). Mutations in STX1B, encoding a presynaptic protein, cause feverassociated epilepsy syndromes. Nat. Genet. 46 (12), 1327-32. doi: 10.1038/ ng. 3130

Speed, D., O'Brien, T. J., Palotie, A., Shkura, K., Marson, A. G., Balding, D. J., et al. (2014). Describing the genetic architecture of epilepsy through heritability analysis. Brain 137 (Pt 10), 2680-2689. doi: 10.1093/brain/awu206

Steinlein, O. K., Mulley, J. C., Propping, P., Wallace, R. H., Phillips, H. A., Sutherland, G. R., et al. (1995). A missense mutation in the neuronal nicotinic acetylcholine receptor alpha 4 subunit is associated with autosomal dominant nocturnal frontal lobe epilepsy. Nat. Genet. 11 (2), 201-203. doi: 10.1038/ ng1095-201

Stucchi, R., Plucinska, G., Hummel, J. J. A., Zahavi, E. E., Guerra San Juan, I., Klykov, O., et al. (2018). Regulation of KIF1A-driven dense core vesicle transport: $\mathrm{Ca}(2+) / \mathrm{CaM}$ controls DCV binding and Liprin-alpha/TANC2 recruits DCVs to postsynaptic sites. Cell Rep. 24 (3), 685-700. doi: 10.1016/ j.celrep.2018.06.071

Wong, M., and Guo, D. (2013). Dendritic spine pathology in epilepsy: cause or consequence? Neuroscience 251, 141-150. doi: 10.1016/..neuroscience.2012.03.048

Yang, Q., Huang, Z., Luo, Y., Zheng, F., Hu, Y., Liu, H., et al. (2019). Inhibition of Nwd1 activity attenuates neuronal hyperexcitability and GluN2B phosphorylation in the hippocampus. EBioMedicine 47, 470-483. doi: 10.1016/j.ebiom.2019.08.050

Yonekawa, Y., Harada, A., Okada, Y., Funakoshi, T., Kanai, Y., Takei, Y., et al. (1998). Defect in synaptic vesicle precursor transport and neuronal cell death in KIF1A motor protein-deficient mice. J. Cell Biol. 141 (2), 431-441. doi: 10.1083/jcb.141.2.431

Zhang, H., Tian, X., Lu, X., Xu, D., Guo, Y., Dong, Z., et al. (2019). TMEM25 modulates neuronal excitability and NMDA receptor subunit NR2B degradation. J. Clin. Invest. 129 (9), 3864-3876. doi: 10.1172/JCI122599

Conflict of Interest: The authors declare that the research was conducted in the absence of any commercial or financial relationships that could be construed as a potential conflict of interest.

Copyright (c) 2020 Guo, Chen, Yang, Xu, Lin, Ma, Chen, Hu, Ma, Wang and Tian. This is an open-access article distributed under the terms of the Creative Commons Attribution License (CC BY). The use, distribution or reproduction in other forums is permitted, provided the original author(s) and the copyright owner(s) are credited and that the original publication in this journal is cited, in accordance with accepted academic practice. No use, distribution or reproduction is permitted which does not comply with these terms. 